\title{
Effect of cigarette smoking on nasal mucociliary clearance and ciliary beat frequency
}

\author{
PJ StANley, R Wilson, MA GREENSTONE, L MacWilliam, PJ COLE \\ From the Host Defence Unit, Department of Medicine, Cardiothoracic Institute, London
}

\begin{abstract}
Despite the in vitro ciliotoxicity of tobacco smoke and the abnormal mucociliary clearance found in smoking related chronic bronchitis, studies of mucociliary clearance in healthy smokers have produced variable results. The nasal mucociliary clearance of saccharin and the in vitro nasal ciliary beat frequency were studied in healthy smokers and non-smokers. One of 29 smokers had a nasal mucociliary clearance time of over 60 minutes; in the remaining 28 the mean (SD) clearance time was $20.8(9.3)$ minutes, which was significantly longer $(p<0.001)$ than the mean time of 11.1 (3.8) minutes in 27 lifelong non-smokers. There was no significant difference between the mean nasal ciliary beat frequency of 10 smokers and 10 non-smokers. There were no significant differences in mean ciliary beat frequency or mean nasal mucociliary clearance time after 10 healthy non-smoking volunteers had smoked two cigarettes each, exhaling the smoke through their nostrils. Unless there is a prompt reversal of any ciliotoxic effect of tobacco smoke when cilia are removed for in vitro examination, the defective clearance seen in chronic cigarette smokers seems unlikely to be due to slowed ciliary beat frequency. It may be due to reduction in number of cilia or to change in the viscoelastic properties of mucus. The failure to detect any acute effect of tobacco smoke is in keeping with this hypothesis.
\end{abstract}

Compounds such as hydrogen cyanide, acrolein, formaldehyde, ammonia, and phenols in tobacco smoke are toxic to mammalian cilia in vitro, ${ }^{1}$ and small quantities of whole smoke or its aqueous extract cause ciliostasis in human respiratory epithelium in vitro. ${ }^{23}$ Patients with chronic bronchitis related to smoking have been shown to have delayed pulmonary mucociliary clearance ${ }^{4}$ but studies in healthy subjects have not shown a consistent difference between smokers and non-smokers. Whole lung clearance, assessed with inhaled radiolabelled particles, has been shown by some workers to be slower in healthy smokers than in non-smokers, ${ }^{56}$ while others report no significant difference between these groups. ${ }^{7}$ Studies of acute exposure to tobacco smoke from one or more cigarettes have produced even more variable results, including no change, ${ }^{8}$ acceleration, ${ }^{9}$ and slowing ${ }^{10}$ of pulmonary clearance, and a variable effect on tracheal mucus velocity. ${ }^{11}$ These discrepancies may be due to differences in technique. There is considerable variation between individuals in measurements of

Address for reprint requests: Dr PJ Cole, Department of Medicine, Cardiothoracic Institute, Brompton Hospital, London SW3 6HP.

Accepted 13 January 1986 whole lung clearance ${ }^{712}$ and factors such as site of particle deposition or amount of coughing may affect the result.

The upper respiratory tract is readily accessible and such factors are more easily controlled during measurement of mucociliary clearance in the nose, although there remains considerable variation between subjects. Ewert ${ }^{13}$ observed slower transport of a dye across a small area of anterior septal mucosa in healthy smokers than in non-smokers, but Quinlan et $a l^{14}$ reported no difference between smokers and non-smokers in nasal clearance of insoluble radiolabelled resin particles placed on the inferior nasal turbinate. Although relatively few subjects were examined in the latter study, the difference in results between the two studies may also relate to the different areas of the nasal mucosa tested and the different indicators used. The timing of the first appearance in the oropharynx of a soluble indicator such as saccharin or dye placed anteriorly in the nose (on the basis of the subject's report of a sweet taste in the case of saccharin) measures the overall fastest flow rate in the nose, whereas the use of larger, insoluble particles demonstrates the considerable variation in flow rates between different areas of the nasal mucosa. 
We have studied nasal mucociliary clearance, using a saccharin test, and in vitro nasal ciliary beat frequency in healthy cigarette smokers and nonsmokers. We have also tested effects of acute exposure to tobacco smoke on these indices in non-smokers.

\section{Methods}

NASAL MUCOCILIARY CLEARANCE

Nasal mucociliary clearance was measured by means of a saccharin method, ${ }^{15}$ as modified by Rutland and Cole. ${ }^{16}$ A $1 \mathrm{~mm}$ diameter particle of saccharin was placed $1 \mathrm{~cm}$ from the anterior end of the inferior nasal turbinate of a nostril verified by inspection not to be obstructed. The subject was requested not to smoke, eat, drink, cough, or sneeze during the test, and was positioned with head flexed $10^{\circ}$. The time from the placing of the particle to the first perception of a sweet taste was recorded in minutes. This test has been shown to be reproducible in individual subjects on different occasions. ${ }^{17}$ The within subject coefficient of variation for nasal mucociliary clearance measured in one healthy subject on 10 different days was $26.8 \%$.

\section{CILIARY BEAT FREQUENCY}

Ciliated epithelium was obtained without local anaesthesia from the inferior nasal turbinate with a cytology brush and transferred to tissue culture medium 199 (Flow laboratories Ltd) ${ }^{18}$ A sealed microscope coverslip slide preparation was made and kept at $37^{\circ} \mathrm{C}$ on a warm stage. Ciliary beat frequency was measured by a photometric technique ${ }^{18}$ and the results were expressed as a mean of 10 readings from areas of epithelium randomly chosen with a graticule. The within subject coefficient of variation for ciliary beat frequency measured in one healthy subject on 10 different days was $8 \%$.

\section{LONG TERM EXPOSURE TO CIGARETTE SMOKE}

Nasal mucociliary clearance was measured in 27 lifelong non-smokers-18 women and nine men with a mean age of 33.1 (range 19-66) years - and in 29 cigarette smokers-17 women and 12 men with a mean age of 28.8 (19-45) years. All smokers had smoked regularly for at least five years; 24 smoked more than 20 cigarettes a day and five 10-19 cigarettes a day (mean pack years 15.7, range 4.5-40). Regular exhalation of the smoke through the nose was reported by 10 smokers; the others rarely or never consciously exhaled smoke by this route. No subject had a history of chronic nasal or chest disease, or had an acute upper respiratory tract infection up to one month before or one week after testing. All tests in smokers were performed between 10.00 and 16.00 hours and after abstension from smoking for at least one hour. In 10 healthy smokers of more than 20 ciga- rettes a day and in 10 healthy non-smokers, nasal cilia were obtained for estimation of ciliary beat frequency from the same nostril at least one week after measurement of nasal mucociliary clearance.

\section{ACUTE EXPOSURE TO CIGARETTE SMOKE}

Nasal mucociliary clearance and ciliary beat frequency were measured in 10 non-smoking volunteers - eight men and two women of mean age 26.8 (range 23-31) years. The following day each subject smoked two medium tar cigarettes consecutively in a small enclosed room, exhaling the smoke slowly through the nose via the pharynx, without deliberate inhalation into the lung, to maximise exposure of the nasal epithelium to the smoke. The ciliary beat frequency of nasal cilia from the same nostril was measured immediately after exposure and nasal mucociliary clearance measured 30 minutes laterafter the mouth had been rinsed with water to avoid reduction in taste perception by the smoke.

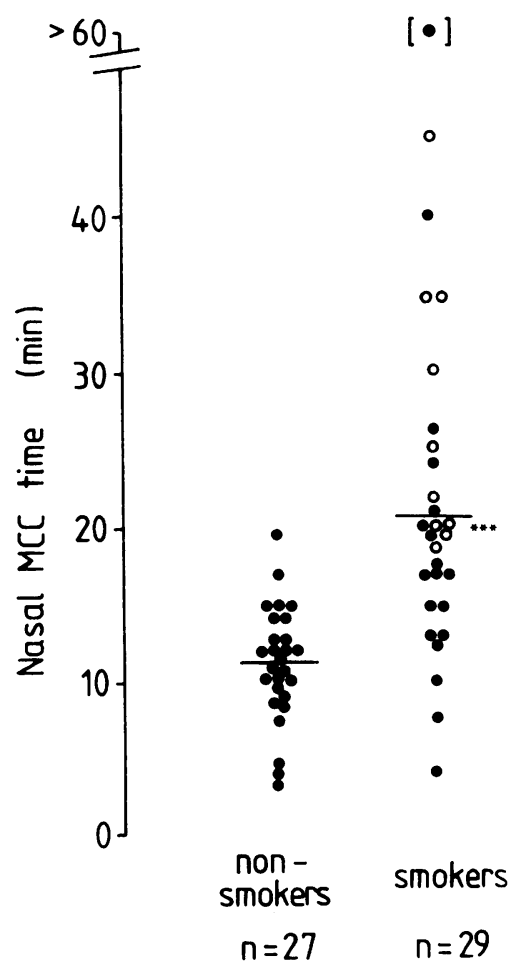

Fig 1 Nasal mucociliary clearance (MCC) times of healthy non-smokers and healthy smokers. The bar represents the mean value excluding the subject with a nasal MCC time greater than 60 minutes. The open circles represent the smokers who regularly exhaled smoke through their noses. ***p $<0.001$ (Mann-Whitney U test). 


\section{Results}

LONG TERM EXPOSURE TO CIGARETTE SMOKE

The results of nasal mucociliary clearance estimations in smokers and non-smokers are shown in figure 1. One smoker of more than 40 cigarettes a day had a clearance time of over 60 minutes. Mean (SD) clearance time in the remaining smokers was $20.8(9.3)$ minutes and in the non-smokers 11.1 (3.8) minutes this difference being significant ( $p<0.001$, MannWhitney U test).

Smokers who regularly exhaled smoke through their noses had a mean nasal mucociliary clearance time of 27.1 (8.9) minutes, which was significantly longer than the mean time of 17.3 (7.7) minutes for smokers who did not regularly exhale by this route (p $<0.05$, Mann-Whitney U test). The mean (SD) nasal ciliary beat frequency was $13.7(1.3) \mathrm{Hz}$ in the 10 smokers and 13.7 (1.4) $\mathrm{Hz}$ in the 10 non-smokersnot significantly different.

\section{ACUTE EXPOSURE TO CIGARETTE SMOKE}

The nasal mucociliary clearance times and nasal ciliary beat frequencies before and after exposure to smoke in the 10 non-smoking healthy subjects are shown in figure 2 . There were no significant differences between the group's mean nasal mucociliary clearance time or mean ciliary beat frequency before smoking (13.4 (4.2) min and $13.7(0.8)$ $\mathrm{Hz}$ and after smoking (13.4 (3.9) min and 14.1 (0.9) $\mathrm{Hz}$ ). The differences between nasal clearance times and ciliary beat frequencies in individuals before and after smoking were within the expected limits of intrasubject variability.

\section{Discussion}

Exposure of the nasal mucosa to toxic products of tobacco smoke is likely to vary considerably between smokers, depending on the number and type of cigarettes smoked and on smoking habits. Most of the smokers we studied smoked a similar number of cigarettes; the only person who smoked more than 40 cigarettes per day was notable in having a grossly prolonged nasal clearance time (greater than 60 minutes). Smokers who exposed their nasal mucosa to more smoke by regular exhalation via the nose had more prolonged nasal mucociliary clearance, even though subjects who do not deliberately exhale by this route will inevitably expose their nasal mucosa to smoke because of lack of awareness of nasal exhalation or "passive" inhalation of their own smoke.

The nasal mucociliary clearance was measured at least one hour after exposure of the nasal mucosa to cigarette smoke, suggesting a chronic slowing of clearance rather than the temporary slowing reported
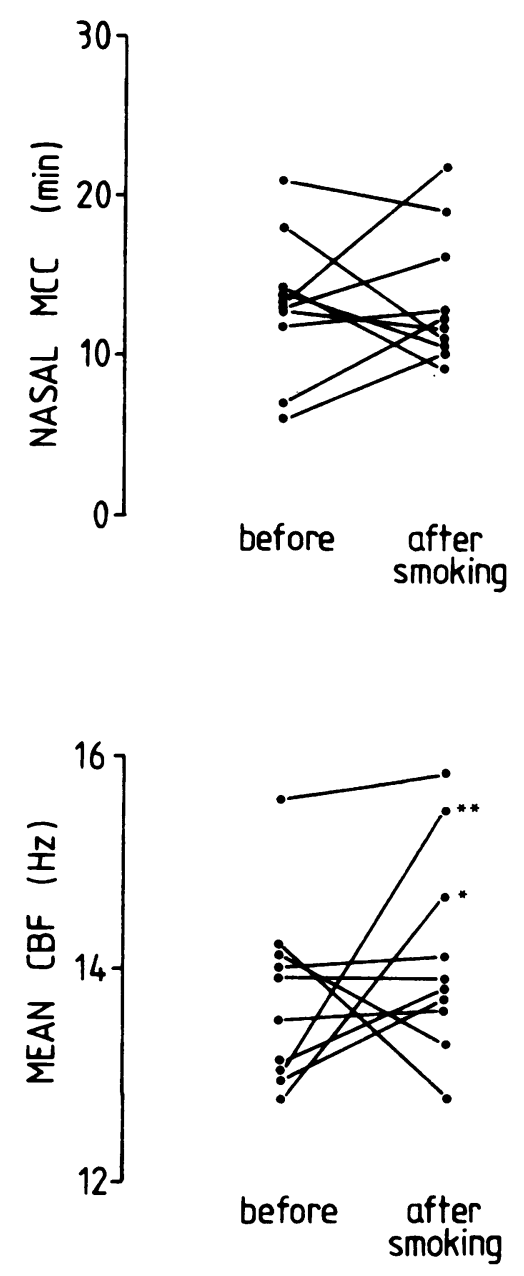

Fig 2 Nasal mucociliary clearance (MCC) times and nasal ciliary beat frequencies (CBF) (mean of 10 readings) in non-smokers before and after they had smoked two cigarettes. ${ }^{*} p<0.05 ;{ }^{* *} p<0.01$ (Students't test).

in studies on the lung. ${ }^{10}$ Reduced nasal mucociliary clearance in regular cigarette smokers with a normal ciliary beat frequency requires explanation. Incoordination of ciliary beating is a possibility, although this was not observed in the epithelial strips during the ciliary beat frequency measurements. Increased areas of non-ciliated epithelium could account for poor clearance and if this was of uneven distribution it would not be detected with the brushing technique described. Such changes have been described at necropsy in the peripheral airways of young smokers (dying of non-respiratory causes without a history of chronic airways obstruction or pathological changes 
of emphysema) by comparison with non-smokers. ${ }^{19}$ Changes in the viscoelastic properties of mucus, which depend largely on the presence of glycoproteins, could be another important factor. Jeffery et $a l^{20}$ showed a change in the granule staining in mucus secreting cells in the airways of rats exposed to tobacco smoke, reflecting a change in the glycoproteins, and Kollerstrom et $a l^{21}$ reported a higher proportion of sulphated mucins relative to sialomucins in the mucus glands of the tracheobronchial tree of smokers at necropsy than in non-smokers. Such a change in glycoprotein composition would, however, be expected to decrease rather than increase mucus viscosity ${ }^{22}$ and have little effect on clearance unless the secretions were too "thin" to be moved by the cilia. Even in the presence of mucus with normal viscoelastic properties, prolonged clearance could be produced by changes in the depth of the periciliary fluid in which the cilia beat-a layer which was too deep causing disconnection of the tips of the cilia from the overlying mucus and too shallow a layer causing entanglement of cilia.

We have not shown any consistent effect of in vivo acute exposure of the nasal mucosa of healthy nonsmokers to smoke from two cigarettes on nasal mucociliary clearance or ciliary beat frequency, although in vitro studies show that smoke from one cigarette blown over fragments of human upper and lower respiratory tract epithelium causes immediate and irreversible ciliostasis. ${ }^{3}$ Thus cilia exposed in situ to tobacco smoke appear to be more resistant to its toxic effects, possibly protected by an intact mucus layer that would be disrupted in the in vitro experiment. Nevertheless, although Ballenger ${ }^{2}$ showed that basic salt solution through which smoke from two cigarettes had been bubbled also caused irreversible ciliostasis of human respiratory epithelium in vitro within five to 28 minutes, when exposure was stopped before cessation of ciliary beating the toxic effect was reversible. Our results do not exclude a slight reduction in ciliary beat frequency in vivo that is reversed within 10 minutes of removal of the strips of ciliated epithelium - this time delay being necessary for specimen preparation. Likewise, a temporary effect of smoking on nasal mucociliary clearance that is reversed within 30 minutes is not excluded by our findings since this interval elapsed between the end of smoking and testing nasal mucociliary clearance, so that any dulling of taste perception by the smoke would not interfere with the saccharin test. Kaminski et $a l^{23}$ found that the reduction in the rate of transport of particles along the trachea of a cat produced by acute exposure to tobacco smoke had almost completely disappeared 7.5 minutes after exposure ceased. Such a short lived effect on nasal mucociliary clearance in man would have little clinical relevance unless the effect were cumulative. Only two cigarettes were smoked in our acute study; smoking more, or the retention of toxic substances within the secretions over longer periods, might conceivably lead to an effect on nasal mucociliary clearance.

The chronic changes in nasal mucociliary clearance we found in regular smokers combined with their normal ciliary beat frequency would be more compatible with reduction in the number of cilia or change in the viscoelastic properties of mucus (or both) than with temporary slowing or incordination of ciliary beating.

PS was supported by Berk Pharmaceuticals, RW by Janssen Pharmaceuticals, MAG by Allen and Hanburys, and LMcW by the Wellcome Trust. This work was supported by the Wellcome Trust.

\section{References}

1 Kensler GJ, Battista SP. Components of cigarette smoke with ciliary depressant activity. $N$ Engl $J$ Med 1963;269:1161-6.

2 Ballenger JJ. Experimental effect of cigarette smoke on human respiratory cilia. $N$ Engl J Med 1960;263:832-5.

3 Dalhamn T. The effect of cigarette smoke on ciliary activity in the upper respiratory tract. Arch Otolaryngol 1959;70:166-7.

4 Pavia D, Bateman JRM, Clarke SW. Deposition and clearance of inhaled particles. Bull Eur Physiopathol Respir 1980;16:335-66.

5 Lourenco RV, Klimek MF, Borowski CJ. Deposition and clearance of $2 \mu$ particles in the tracheobronchial tree of normal subjects-smokers and non-smokers. J Clin Invest 1971;50:1411-20.

6 Camner P, Philipson K. Tracheobronchial clearance in smoking discordant twins. Arch Environ Health 1972;25:60-3.

7 Pavia D, Short MD, Thomson ML. No demonstrable long-term effects of cigarette smoking on the mucociliary mechanism of the human lung. Nature 1970;226:1228-31.

8 Yeates DB, Aspin N, Levison H, Jones MT, Bryan AC. Mucociliary tracheal transport rates in man. $J$ Appl Physiol 1973;39:487-95.

9 Albert RE, Peterson HT, Bohning DE. Short-term effects of cigarette smoking on bronchial clearance in humans. Arch Environ Health 1975;30:361-7.

10 Pavia D, Thomson ML, Pocock SJ. Evidence for temporary slowing of mucociliary clearance in the lung by tobacco smoke. Nature 1971;231:325-6.

11 Yergin B, Goodman RM, Landa JF, Golinvaux MK, Sackner MA. Effects of cigarette smoking on tracheal mucous velocity in young smokers with and without small airways obstruction. Am Rev Resp Dis (Suppl) 1977;115:183.

12 Camner P, Philipson K, Friberg L. Human tracheobronchial clearance studies. Arch Environ Hlth 1971;22: 444-9.

13 Ewert G. On the mucus flow rate in the human nose Acta Otolaryngol (Stockh) (Suppl) 1965;200:1-65.

14 Quinlan MF, Salman SD, Swift DL, Wagner HN, Proc- 
tor DF. Measurement of mucociliary function in man. Am Rev Resp Dis 1969;99:13-23.

15 Andersen I, Camner P, Jensen PL, Philipson K, Proctor DF. A comparison of nasal and tracheobronchial clearance. Arch Environ Health 1974;29:290-3.

16 Rutland J, Cole PJ. Nasal mucociliary clearance and ciliary beat frequency in cystic fibrosis compared to sinusitis and bronchiectasis. Thorax 1981;36:654-8.

17 Stanley P, MacWilliam L, Greenstone M, Mackay I, Cole P. Efficacy of a saccharin test for screening to detect abnormal mucociliary clearance. $\mathrm{Br} J$ Dis Chest 1984;78:62-5.

18 Rutland J, Cole PJ. Non-invasive sampling of nasal cilia for measurement of beat frequency and study of ultrastructure. Lancet 1980;ii:564-5.

19 Niewoehner DE, Kleinerman J, Rice DB. Pathologic changes in the peripheral airways of young smokers. $N$ Engl J Med 1974;291:755-8.

20 Jeffery PK, Reid LM. The effect of tobacco smoke, with and without phenylmethyloxadiazole (PMO), on rat bronchial epithelium: a light and electron microscopic study. J Pathol 1981;133:341-59.

21 Kollerstrom N, Lord PW, Whimster WF. A difference in the composition of bronchial mucus between smokers and non-smokers. Thorax 1977;32:155-9.

22 Lopez-Vidriero MT, Reid L. Bronchial mucus in health and disease. Br Med Bull 1978;34:63-74.

23 Kaminski EJ, Fancher OE, Calandra JC. In vivo studies of the ciliastatic effects of tobacco smoke. Absorption of ciliastatic components by wet surfaces. Arch Environ Health 1968;16:188-93. 